\title{
Synergistic sequential enhancement of docetaxel
}

$\mathrm{T}$ he efficacy of the microtubule inhibitor docetaxel is markedly increased by co-administration with an analog of noscapine, a naturally occurring nontoxic plant alkaloid. Translation of this positive outcome of cell-based and animal experiments to the clinic would improve the quality of life of thousands of men with metastatic castration-resistant prostate cancer.

Until very recently, patients with metastatic castration-resistant prostate cancer could be offered palliative care only. In 2010, docetaxel was one of two agents with life-extending potential approved for use in this population (the other was sipuleucel-T immunotherapy). Docetaxel-a tubulin-binding drug-has become standard first-line therapy for men whose advanced prostate cancer is unresponsive to androgen ablation.

Now patients face a difficult decision: is the chance to modestly prolong survival worth the impairment of quality of life associated with docetaxel treatment? The drug-like many systemic chemotherapies-is usually administered at its maximum tolerated dose. Such high doses are associated with debilitating toxicities, and necessitate interrupted dosing schedules. Tumor regrowth is common during these 'rest periods'. As a result, near-complete cure is rarely achieved.

Researchers from Georgia State University in Atlanta believe that many of these issues could be overcome by leveraging synergistic interactions between two drugs. Ritu Aneja and colleagues have shown that a combined regimen of docetaxel and another microtubule inhibitor (the noscapine analog EM011) inhibits tumor growth to a much greater extent than either drug alone.

Nude mice harboring tumors composed of human androgen-independent PC-3 cells were treated for 5 weeks. Treatment comprised one infusion of docetaxel ( $5 \mathrm{mg} / \mathrm{kg}$ body weight) per week, plus daily oral administration of EM011 $(300 \mathrm{mg} / \mathrm{kg}$ $\underset{\alpha \beta}{\infty}$

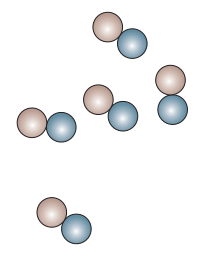

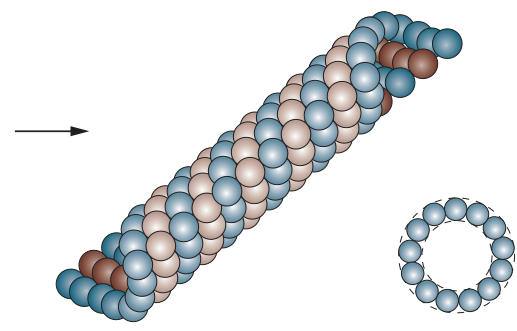

body weight). This regimen resulted in an $89 \%$ reduction in tumor volume, relative to untreated controls. The tumors of animals that received docetaxel or EM011 only shrank by $64 \%$ and $35 \%$, respectively.

Immunostaining of tumor samples from the treated mice indicated that the superiority of the combinatorial strategy was a function of enhanced apoptosis as well as inhibited cellular proliferation. Importantly, the body weight of animals that received both drugs did not drop significantly over the 5-week study period, indicating that the dual-drug regimen was well tolerated.

Aneja et al. assert that these findings present a "unique opportunity to reduce the dose-levels of docetaxel well below its maximum tolerated doses by compensating it with daily oral EM011 doses in prostate cancer therapy". Their work, written up in Biochemical Pharmacology, also indicates the importance of the relative timings of administration of different agents in multidrug regimens.

In vitro assays showed that docetaxeltreated PC-3 cells exited mitosis after $36 \mathrm{~h}$, making them less susceptible to apoptosis. Adding EM011 to culture media $12 \mathrm{~h}$ after addition of docetaxel maximally enhanced the number of cells arrested in the mitotic phase, during which they are most likely to undergo programmed cell death.

A similar temporal relationship has been noted by a Korean team studying docetaxel, this time in combination with histone deacetylase (HDAC) inhibitors. Sequential treatment of androgen-independent DU145 cells with docetaxel followed by a HDAC inhibitor maximized synergistic cytotoxicity. Simultaneous administration of the two drugs, or a 'reverse sequence' of an HDAC inhibitor followed by docetaxel, was much less effective.

The combination of docetaxel and the HDAC blocker PXD101 shrank tumors in DU145 xenografted animals to the same degree as a double dose of docetaxel. Again, arrest of cells in an apoptosis-susceptible phase of the cell cycle was noted. Enhancement of programmed cell death, indicated by modulation of expression of $\mathrm{Bcl}-2$ proteins, was a key mechanism underlying the synergistic effect.

HDAC inhibitors have long been used in psychiatry and neurology as mood stabilizers and anti-epileptics. During the past 5 years, two drugs of this class-vorinostat and romidepsin-have been approved by the FDA for the treatment of cutaneous T-cell lymphoma. Currently, a phase II trial of the oral HDAC inhibitor SB939 in castrationresistant prostate cancer is underway in Canada. Incorporating drugs with a longstanding history of clinical use, such as HDAC blockers, into docetaxel-based protocols for advanced prostate cancer could maximize quality of life without compromising clinical efficacy.

Suzanne J. Farley

\footnotetext{
Original articles Pannu, V. et al. Synergistic antimicrotubule therapy for prostate cancer. Biochem. Pharmacol. doi:10.1016/j.bcp.2010.11.006 | Hwang, J. J. et al. Histone deacetylase inhibitor potentiaties anticancer effect of docetaxel via modulation of $\mathrm{Bcl}-2$ family proteins and tubulin in hormone refractory prostate cancer cells. J. Urol. 184, 2557-2564 (2010)
} 